\title{
Experimental demonstration of a BDCZ quantum repeater node
}

\author{
Zhen-Sheng Yuan $^{1,2 *}$, Yu-Ao Chen ${ }^{1,2 *}$, Bo Zhao ${ }^{1}$, Shuai Chen ${ }^{1}$, Jörg Schmiedmayer ${ }^{3}$ \& Jian-Wei Pan ${ }^{1,2}$ \\ ${ }^{1}$ Physikalisches Institut, Ruprecht-Karls-Universität Heidelberg, Philosophenweg 12, 69120 Heidelberg, Germany \\ ${ }^{2}$ Hefei National Laboratory for Physical Sciences at Microscale and Department of Modern Physics, \\ University of Science and Technology of China, Hefei, Anhui 230026, China \\ ${ }^{3}$ Atominstitut der Österreichischen Universitäten, TU-Wien, A-1020 Vienna Austria \\ * These authors contributed equally to this work.
}

Quantum communication is a method that offers efficient and secure ways for the exchange of information in a network. Large-scale quantum communication $[1,2,3,4]$ (of the order of 100 km) has been achieved; however, serious problems occur beyond this distance scale, mainly due to inevitable photon loss in the transmission channel. Quantum communication eventually fails [5] when the probability of a dark count in the photon detectors becomes comparable to the probability that a photon is correctly detected. To overcome this problem, Briegel, Dür, Cirac and Zoller (BDCZ) introduced the concept of quantum repeaters [6], combining entanglement swapping [7] and quantum memory to efficiently extend the achievable distances. Although entanglement swapping has been experimentally demonstrated [8], the implementation of BDCZ quantum repeaters has proved challenging owing to the difficulty of integrating a quantum memory. Here we realize entanglement swapping with storage and retrieval of light, a building block of the BDCZ quantum repeater. We follow a scheme [9, 10] that incorporates the strategy of BDCZ with atomic quantum memories [11]. Two atomic ensembles, each originally entangled with a single emitted photon, are projected into an entangled state by performing a joint Bell state measurement on the two single photons after they have passed through a 300-m fibre-based communication channel. The entanglement is stored in the atomic ensembles and later verified by converting the atomic excitations into photons. Our method is intrinsically phase insensitive and establishes the essential element needed to realize quantum repeaters with stationary atomic qubits as quantum memories and flying photonic qubits as quantum messengers.

Although the BDCZ protocol [6] attracted much interest as a solution to extend the communication length, the absence of quantum memory has hindered the implementation of quantum repeaters. In 2001, Duan, Lukin, Cirac and Zoller (DLCZ) proposed an alternative quantum repeater scheme [11] where linear optics and atomic ensembles are used to incorporate entanglement connection and quantum memory into a single unit. Motivated by the DLCZ protocol, number-state entanglement between two atomic ensembles has been observed [12, 13]. Most recently, a functional quantum node [14] based on asynchronous preparation of number-state entanglement for two pairs of atomic ensembles - the basic element of the DLCZ protocol - has also been demonstrated.

However, two serious drawbacks make the DLCZ-type functional quantum nodes [11, 14] unlikely to be a realistic solution for long-distance quantum communication [9, 10, 15. First, the required long-term subwavelength stability of the path difference between two arms of a large scale single-photon interferometer spanning the whole communication distance is very difficult to achieve 9, 10], even with the latest and most sophisticated technology for coherent optical phase transfer [16]. Second, the swapping of number-state entanglement using a single-photon interferometer leads to the growth of a vacuum component in the generated state, and to the rapid growth of errors due to multiple emissions from individual ensembles [15].

A novel solution [9, 10], is to combine the atomic quantum memory in DLCZ and the strategy of BDCZ. Since in this scheme two-photon interference is used to generate long-distance entanglement, the stability requirement for the path differences is determined by the coherence length of the photons and is consequently 7 orders of magnitude looser [17] than in the DLCZ protocol. Moreover, the vacuum component can be suppressed and is no longer a dominant term after a few entanglement connections [9, 10]. Following this scheme, we demonstrated the implementation of a quantum repeater node, involving entanglement swapping with the function of storage and retrieval of light. A high precision of local operations has been achieved that surpasses the theoretical threshold [6] required for the realization of robust quantum repeaters for long-distance quantum communication.

In our experiment, to demonstrate entanglement swapping with storage and retrieval of light, we follow three steps: implementing two atom-photon entanglement sources, sending the flying qubits (the photons) to an intermediate station for a BSM, and verifying the entanglement between the stationary qubits (the two remote atomic ensembles).

Unlike previous atom-photon entanglement sources realized with trapped ions [18], single atoms in a cavity [19], or two spatially separated atomic ensembles 20], we use 


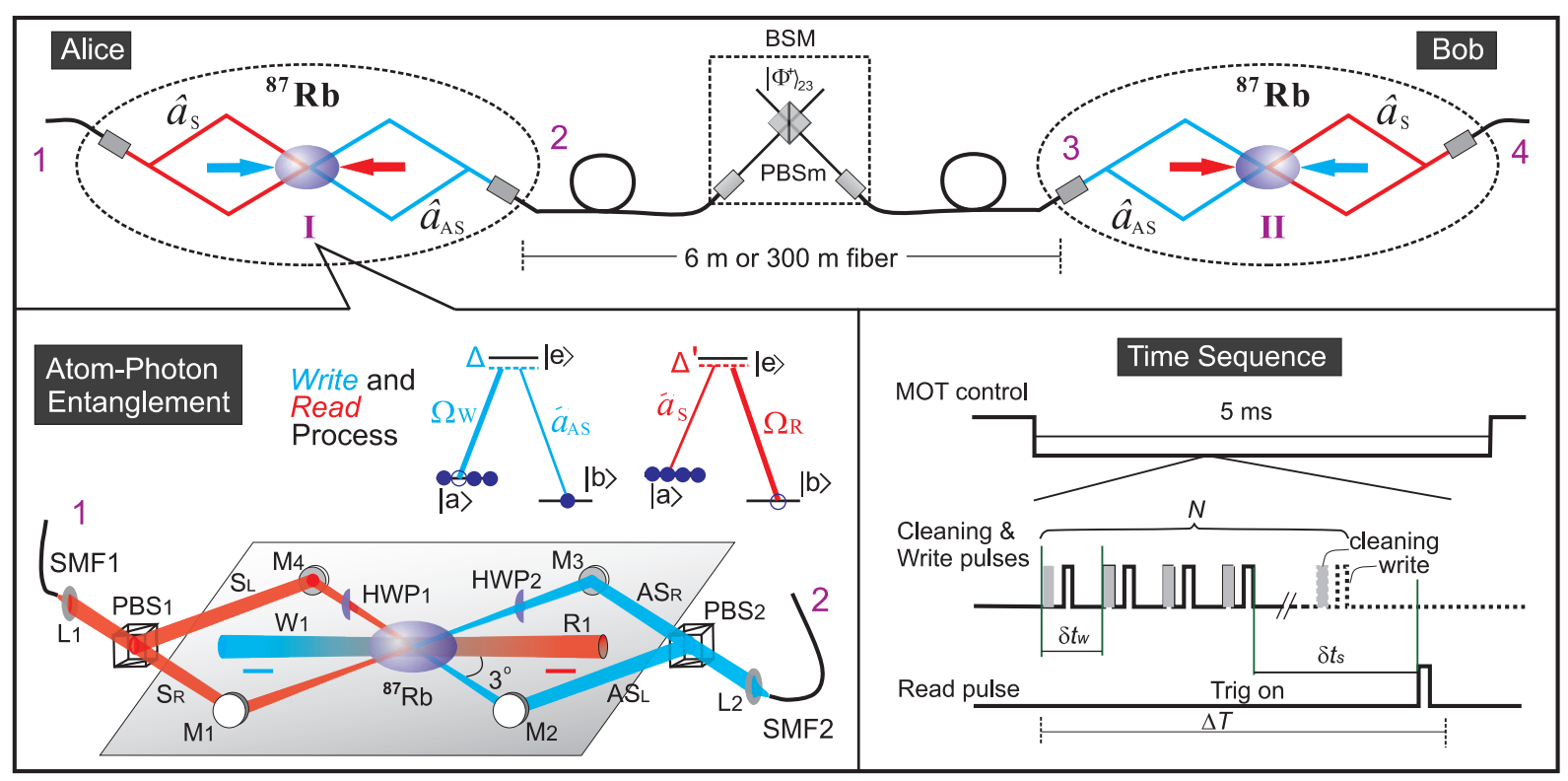

FIG. 1: The experimental scheme for entanglement swapping. Upper Panel: photons 2 and 3 overlap at BSM and are projected to the state $\left|\Phi^{+}\right\rangle_{2,3}$ through which the entanglement is generated between the two atomic ensembles I and II confined by magneto-optical traps (MOTs) in different glass cells separated by $\sim 60 \mathrm{~cm}$. Here 1, 2, 3 and 4 indicate four photons emerging from the anti-Stokes modes $\left(\hat{a}_{A S}\right)$ and Stokes modes $\left(\hat{a}_{S}\right)$, and $\left|\Phi^{+}\right\rangle_{2,3}$ is one of the four Bell states. Lower-left Panel: atomphoton entanglement. Shown are energy levels $\{|a\rangle,|b\rangle,|e\rangle\}=\left\{\left|5 S_{1 / 2}, F=2\right\rangle,\left|5 S_{1 / 2}, F=1\right\rangle,\left|5 P_{1 / 2}, F=2\right\rangle\right\}$ and the configuration of light beams. PBS, polarizing beam splitter; HWP, half-wave plate; L, lens; M, mirror; SMF, single-mode fibre; W, write beam; R, read beam; S, Stokes field; AS, anti-Stokes field; $\Omega$, Rabi frequency of light fields. Lower-right Panel: The time sequence of the experimental procedure at each site. For 6-m (300-m) fibre connection, there are 250 (200) experiment cycles in $5 \mathrm{~ms}$ and $\Delta T$ is $16 \mu \mathrm{s}(20 \mu \mathrm{s})$ for one cycle, which contains $N=10(N=8)$ write sequences. The interval between two neighbouring write pulses is $\delta t_{w}=1 \mu \mathrm{s}(1.5 \mu \mathrm{s})$ and $\delta t_{s}$ is the storage time. Whenever there is a desired coincidence event between photons 2 and 3, the following write sequence is stopped by a feedback circuit and the retrieve process can be started (at the time point labelled 'Trig.on').

two collective excitations in different spatial modes of a single atomic ensemble to implement the atom-photon entanglement [21]. In contrast to the method in which two separated spatial regions in one atomic cloud are covered by their own read and write beams [14], here the two excitation modes share the same write and read beams, which offers high-quality entanglement and longterm stability.

The basic principle is shown in Fig. 1 (see Methods). Alice and Bob each have a cold atomic ensemble consisting of about $10^{8}{ }^{87} \mathrm{Rb}$ loaded by magneto-optical traps (MOTs). At each site atoms are first prepared in the initial state $|a\rangle$, followed by a weak write pulse. Two anti-Stokes fields $A S_{L}$ and $A S_{R}$ induced by the write beam via spontaneous Raman scattering are collected at $\pm 3^{\circ}$ relative to the propagating direction of the write beam. This defines two spatial modes of excitation in the atomic ensembles $(L$ and $R$ ), which constitute our memory qubit.

The two anti-Stokes fields in modes $L$ and $R$ are adjusted to have equal excitation probability and orthogonal polarizations. The two fields are then overlapped at a polarizing beam splitter PBS2 and coupled into a single- mode fiber. Neglecting the vacuum state and higher order excitations, the entangled state between the atomic and photonic qubits can be described effectively as,

$$
|\Psi\rangle_{\text {at-ph }}=\frac{1}{\sqrt{2}}\left(|H\rangle|R\rangle+e^{i \phi_{1}}|V\rangle|L\rangle\right)
$$

where $|H\rangle /|V\rangle$ denotes horizontal/vertical polarization of the single anti-Stokes photon and $|L\rangle /|R\rangle$ denotes single collective excitation in ensemble $L / R, \phi_{1}$ is the propagating phase difference between the two anti-Stokes fields before they overlap at PBS2. Physically, the atomphoton entangled state (10) is equivalent to the maximally polarization-entangled state generated by spontaneous parametric down-conversion [22].

In this way, one can implement two separate and remote atom-photon entanglement sources at Alice (I) and Bob's (II) sites respectively. To make the higher order excitations negligible, a low excitation probability $\left(\chi_{m} \sim 0.01\right)$ is chosen. Due to the imperfect coupling of light modes, the transmission loss, and the inefficiency of single photon detectors, the overall detection efficiency of an emerging anti-Stokes photon $\left(\eta_{\mathrm{AS}}\right)$ is around $25 \%$. To check the quality of atom-photon entanglement, a read 


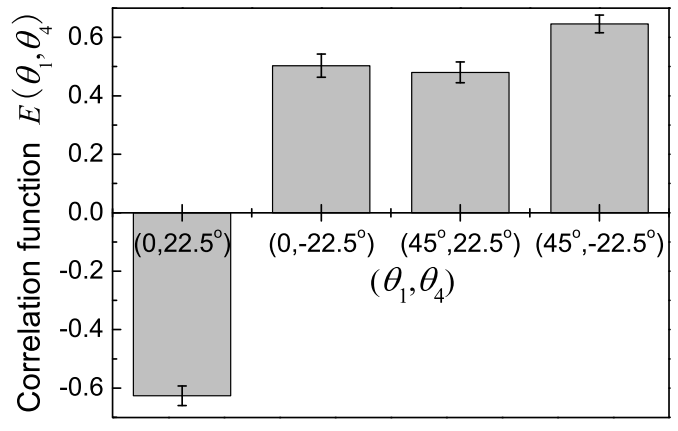

FIG. 2: Correlation functions of a CHSH-type Bell's inequality with the storage time $\delta t_{s}=500 \mathrm{~ns}$. Error bars represent statistical errors, which are \pm 1 standard deviation.

pulse (see Methods) is applied after a controllable timedelay $\delta t_{s}$ to convert the atomic collective excitation back into a Stokes field. Ideally, the retrieve efficiency of the Stokes fields should reach unity. However, various imperfections such as low optical depth of the atomic ensembles and mode mismatching between the write and read pulses lead to a $35 \%$ retrieve efficiency. Together with the non-ideal collection and detection efficiency $(\sim 40 \%)$ of single photon detectors, the overall detection efficiency of the Stokes photon is around $15 \%$. After combining the two retrieved Stokes fields on PBS1 (see Fig. 1), the antiStokes and Stokes fields are in the following maximally polarization-entangled state

$$
|\Psi\rangle_{\mathrm{AS}, \mathrm{S}}=\frac{1}{\sqrt{2}}\left(|H\rangle_{\mathrm{AS}}|H\rangle_{\mathrm{S}}+e^{i\left(\phi_{1}+\phi_{2}\right)}|V\rangle_{\mathrm{AS}}|V\rangle_{\mathrm{S}}\right)
$$

where $\phi_{2}$ represents the propagating phase difference between two Stokes fields before they overlap at PBS1. In our experiment, the total phase $\phi_{1}+\phi_{2}$ is actively stabilized via the built-in Mach-Zehnder interferometer and fixed to zero (see the online supplementary information). With a time-delay $\delta t_{s}=1 \mu \mathrm{s}$, the measured polarization correlations of the Stokes and anti-Stokes photons show a strong violation of a CHSH-type Bell's inequality, with a visibility of $92 \%$, confirming the high quality of our atom-photon entanglement sources. Further measurement shows our atom-photon entanglement still survives up to a storage time of $\delta t_{s}=20 \mu \mathrm{s}$ (see the online supplementary information).

We now describe the entanglement generation between atomic ensembles I and II via entanglement swapping. As shown in Fig. 1 photon 2 from Alice and photon 3 from Bob are both sent through a $3 \mathrm{~m}$ optical fiber to an intermediate station for a joint BSM. In the experiment, we chose to analyze the projection onto the Bell state $\left|\Phi^{+}\right\rangle_{2,3}=\frac{1}{\sqrt{2}}\left(|H\rangle_{2}|H\rangle_{3}+|V\rangle_{2}|V\rangle_{3}\right)$, which is achieved

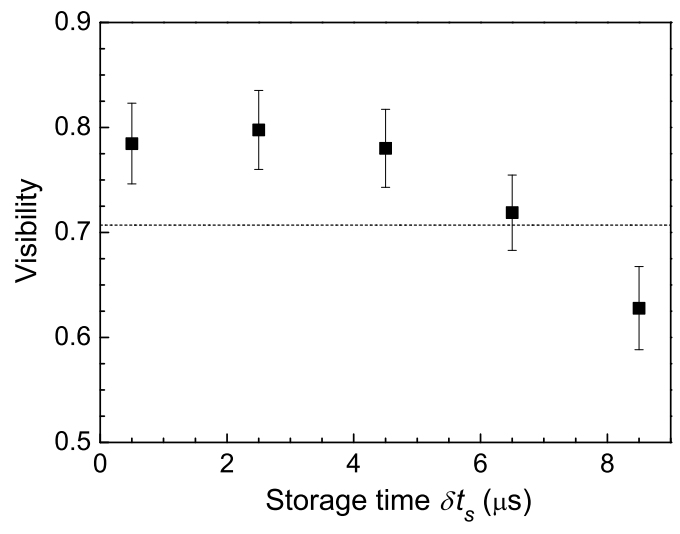

FIG. 3: Visibility as a function of the storage time with 6 $\mathrm{m}$ fiber connection. Black dots are for the visibility and the dashed line shows the threshold for the violation of the CHSHtype Bell's inequality. Error bars represent statistical errors, which are \pm 1 standard deviation.

by overlapping photons 2 and 3 onto a polarizing beam splitter (PBSm) and performing a proper polarization decomposition in the output modes and a subsequent coincidence detection [23]. Conditioned on detecting a $\left|\Phi^{+}\right\rangle_{2,3}$ state at the intermediate station, the two remote atomic ensembles is projected onto an identical entangled state $\left|\phi^{+}\right\rangle_{\mathrm{I}, \mathrm{II}}=\frac{1}{\sqrt{2}}\left(|R\rangle_{\mathrm{I}}|R\rangle_{\mathrm{II}}+|L\rangle_{\mathrm{I}}|L\rangle_{\mathrm{II}}\right)[7,[8]$.

It is worth to note that double excitations in either atomic ensemble I or II will cause false events in the BSM [9, 10], which reduce the success probability of entanglement swapping by a factor of 2 . Experimentally, the false events can be eliminated at the stage of entanglement verification by the four-fold coincidence measurement of photons 1, 2, 3 and 4 . Note that, the detection time of photons 1 and 4 is later than that of photons 2 and 3 by an interval $\delta t_{s}$, the storage time in quantum memories. More importantly, such false events do not affect the applications of our experimental method in quantum repeaters, since the generation of entanglement will be deterministic after a second step of connecting two such nodes, where double excitations are excluded automatically [9, 10].

The established entanglement between atomic ensembles I and II can be verified by converting the atomic spins into an entangled photon pair 1 and 4 , which are in the state $\left|\Phi^{+}\right\rangle_{1,4}$. Here we measure the $S$ parameter in a CHSH-type Bell's inequality,

$$
S=\left|E\left(\theta_{1}, \theta_{4}\right)-E\left(\theta_{1}, \theta_{4}^{\prime}\right)-E\left(\theta_{1}^{\prime}, \theta_{4}\right)-E\left(\theta_{1}^{\prime}, \theta_{4}^{\prime}\right)\right|,
$$

where $E\left(\theta_{1}, \theta_{4}\right)$ is the correlation function and, $\theta_{1}$ and $\theta_{1}^{\prime}$ $\left(\theta_{4}\right.$ and $\left.\theta_{4}^{\prime}\right)$ are the measured polarization bases of photon 1 (4). In the measurement, the polarization settings 


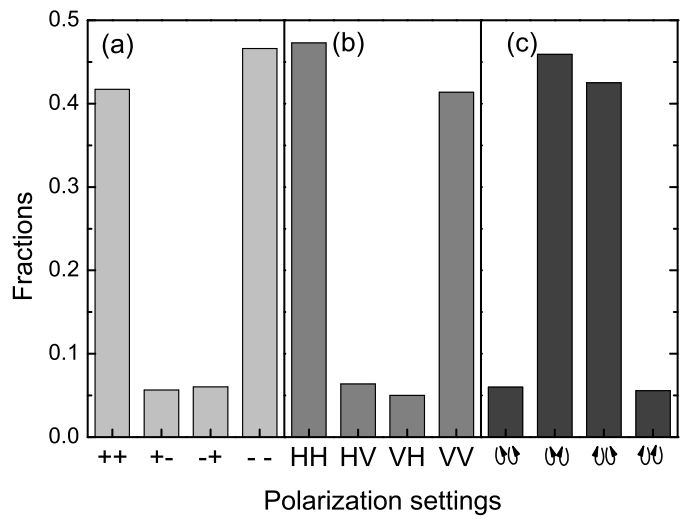

FIG. 4: Experimental outcomes of the fractions at different polarization settings with $300 \mathrm{~m}$ fiber connection. The polarization bases are chosen as (a) $|+\rangle$ and $|-\rangle$, (b) $|H\rangle$ and $|V\rangle$, and (c) $|\circlearrowleft\rangle$ and $|\circlearrowright\rangle$ respectively.

are $\left(0^{\circ}, 22.5^{\circ}\right),\left(0^{\circ},-22.5^{\circ}\right),\left(45^{\circ}, 22.5^{\circ}\right)$ and $\left(45^{\circ},-22.5^{\circ}\right)$, respectively.

At a storage time $\delta t_{s}=500 \mathrm{~ns}$, the measured correlation functions (shown in Fig. 2) result in $S=2.26 \pm 0.07$, which violates Bell's inequality by 3 standard deviations. To observe the lifetime of the entanglement between two remote memory qubits, we measure the interference visibility of photons 1 and 4 as a function of the storage time by choosing the polarization basis of $+/-$ (shown in Fig[3, with $|+\rangle=\frac{1}{\sqrt{2}}(|H\rangle+|V\rangle)$ and $|-\rangle=\frac{1}{\sqrt{2}}(|H\rangle-|V\rangle)$ ). Up to a storage time of $4.5 \mu \mathrm{s}$, the visibility is still higher than the threshold $1 / \sqrt{2}$, sufficient for the violation of Bell's inequality. From the visibilities of the atom-photon and atom-atom entanglements, the precision of local operations at the BSM station is estimated to be better than $97 \%$ (see the online supplementary information). We emphasize that this precision achieved here surpasses the threshold of $95 \%$ for local operations of independent photons necessary for future entanglement purification and connections [6], and therefore fits the requirement for a scalable quantum network.

To demonstrate the robustness of our protocol in generation of quantum entanglement between two atomic ensembles over large distances, we change the length of the two connecting fibers from $3 \mathrm{~m}$ to $150 \mathrm{~m}$. The anti-Stokes photon is delayed $730 \mathrm{~ns}$ and the connection length between Alice and Bob is $300 \mathrm{~m}$. The entanglement swapping can be quantified by the fidelity of the measured state of the atomic ensembles. To determine the fidelity, we write the density matrix of $\left|\phi^{+}\right\rangle_{\mathrm{I}, \mathrm{II}}$ in terms of the Pauli matrices:

$$
\left|\phi^{+}\right\rangle\left\langle\left.\phi^{+}\right|_{\mathrm{I}, \mathrm{II}}=\frac{1}{4}\left(I+\hat{\sigma}_{x} \hat{\sigma}_{x}-\hat{\sigma}_{y} \hat{\sigma}_{y}+\hat{\sigma}_{z} \hat{\sigma}_{z}\right)\right.
$$

Here $\sigma_{x}=|+\rangle\langle+|-|-\rangle\left\langle-\left|, \sigma_{y}=\right| \circlearrowright\right\rangle\langle\circlearrowright|-| \circlearrowleft\rangle\langle\circlearrowleft|$, and $\sigma_{z}=|H\rangle\langle H|-| V\rangle\langle V|$, with $|\circlearrowleft\rangle=(1 / \sqrt{2})(|H\rangle+i|V\rangle)$ and $|\circlearrowright\rangle=(1 / \sqrt{2})(|H\rangle-i|V\rangle)$. After a storage time of $1230 \mathrm{~ns}$ (with a $730 \mathrm{~ns}$ delay being taken into account), the two retrieved photons 1 and 4 are sent to their own polarization analyzer. Three series of polarization settings are used and the measured local observables are shown in Fig. 4. The fidelity of final state $\rho_{\exp }$ on $\left|\phi^{+}\right\rangle_{\mathrm{I}, \mathrm{II}}$ is given by $F=\operatorname{Tr}\left(\rho_{\text {exp }}\left|\phi^{+}\right\rangle\left\langle\left.\phi^{+}\right|_{\mathrm{I}, \mathrm{II}}\right)=0.83 \pm 0.02\right.$, with 2.5 standard deviations beyond the threshold of 0.78 to violate the CHSH-type Bell's inequality for Werner states, demonstrating the success of entanglement swapping. This fidelity is comparable to the average value achieved in the DLCZ-type functional quantum node [14.

In summary, we have successfully demonstrated high precision entanglement swapping with storage and retrieval of light, a building block for quantum repeaters. The extension of our work to longer chains will involve many quantum repeater nodes. To achieve this ambitious goal, several quantities - such as the lifetime and retrieve efficiency of the quantum memory, the fidelity and generation rate of the entanglement state - still need to be improved significantly. We suggest three ways forward. First, better compensation of the residual magnetic field and trapping the atoms in "clock states" 24] with a blue-detuned optical trap 25] should improve the lifetime to $\sim 1 \mathrm{~s}$. Second, a high optical density of the atomic cloud, achieved by the help of traps or by coupling the atoms into an optical cavity [26], should increase the retrieve efficiency close to unity. These improvements of the quantum memory would greatly enhance the fidelity and generation rate of the entanglement. Last, by local generation of entangled pairs of atomic excitations together with the present technique of entanglement swapping, the entanglement distribution rate can be greatly improved [27]. Not only does our work enable immediate experimental investigations of various quantum information protocols, but - with the abovementioned future improvements - entanglement swapping with storage and retrieval of light would also open the way to long-distance quantum communication.

\section{Methods}

As shown in Fig. 1, Alice and Bob each have a cold atomic ensemble consisting of about $10^{8}$ atoms of ${ }^{87} \mathrm{Rb}$ with temperature $\sim 100 \mu \mathrm{K}$. After $20 \mathrm{~ms}$ of loading atoms into their MOTs separated by $\sim 60 \mathrm{~cm}$, we switch off the laser beams and magnetic fields of the MOTs and start a 5-ms-long experiment cycle. At each site, atoms are first prepared in the initial $|a\rangle$, followed by a (50 ns long, $\sim 1 \mu \mathrm{W}$ ) weak write pulse, which has a beam waist of $240 \mu \mathrm{m}$ and is $10 \mathrm{MHz}$ red-detuned from the $|a\rangle \rightarrow|e\rangle$ transition. Two anti-Stokes fields $A S_{L}$ and $A S_{R}$ induced by the write beam via spontaneous Raman scattering are 
collected at $\pm 3^{\circ}$ relative to the propagating direction of the write beam $(70 \mu \mathrm{m}$ waist, $|e\rangle \rightarrow|b\rangle)$. The excitation probability $\left(\chi_{m}\right)$ of the collective modes $m(m=L, R)$ is low $\left(\chi_{m} \ll 1\right)$; thus the state of the atom-photon field can be expressed as [1]

$$
|\Psi\rangle_{m} \sim\left|0_{\mathrm{AS}} 0_{b}\right\rangle_{m}+\sqrt{\chi_{m}}\left|1_{\mathrm{AS}} 1_{b}\right\rangle_{m}+O\left(\chi_{m}\right),
$$

and $\left|i_{\mathrm{AS}} i_{b}\right\rangle_{m}$ denote the $i$-fold excitation of the antiStokes field and the collective spin in the atomic ensemble. The read beam is counter-propagating and modematched with the write beam with a pulse length of 50 ns, a power of $60 \mu \mathrm{W}$ and a frequency close to resonance of the $|b\rangle \rightarrow|e\rangle$ transition.

[1] Peng, C.-Z. et al. Experimental long-distance decoy-state quantum key distribution based on polarization encoding. Phys. Rev. Lett. 98, 010505 (2007).

[2] Rosenberg, D. et al. Long-distance decoy-state quantum key distribution in optical fiber. Phys. Rev. Lett. 98, 010503 (2007).

[3] Schmitt-Manderbach, T. et al. Experimental demonstration of free-space decoy-state quantum key distribution over 144 km. Phys. Rev. Lett. 98, 010504 (2007).

[4] Ursin, R. et al. Entanglement-based quantum communication over $144 \mathrm{~km}$. Nature Physics 3, 481-486 (2007).

[5] Gisin, N., Ribordy, G., Tittel, W. \& Zbinden, H. Quantum cryptography. Rev. Mod. Phys. 74, 145-195 (2002).

[6] Briegel, H.-J., Dür, W., Cirac, J. I. \& Zoller, P. Quantum repeaters: The role of imperfect local operations in quantum communication. Phys. Rev. Lett. 81, 5932-5935 (1998).

[7] Żukowski, M., Zeilinger, A., Horne, M. A. \& Ekert, A. K. "event-ready-detectors" bell experiment via entanglement swapping. Phys. Rev. Lett. 71, 4287-4290 (1993).

[8] Pan, J.-W., Bouwmeester, D., Weinfurter, H. \& Zeilinger, A. Experimental entanglement swapping: Entangling photons that never interacted. Phys. Rev. Lett. 80, 38913894 (1998).

[9] Zhao, B., Chen, Z.-B., Chen, Y.-A., Schmiedmayer, J. \& Pan, J.-W. Robust long-distance quantum communication with atomic ensembles and linear optics. Phys. Rev. Lett. 98, 240502 (2007).

[10] Chen, Z.-B., Zhao, B., Chen, Y.-A., Schmiedmayer, J. \& Pan, J.-W. Fault-tolerant quantum repeater with atomic ensembles and linear optics. Phys. Rev. A 76, 022329 (2007).

[11] Duan, L.-M., Lukin, M. D., Cirac, J. I. \& Zoller, P. Longdistance quantum communication with atomic ensembles and linear optics. Nature (London) 414, 413-418 (2001).

[12] Matsukevich, D. N. \& Kuzmich, A. Quantum state transfer between matter and light. Science 306, 663-666 (2004).

[13] Chou, C.-W. et al. Measurement-induced entanglement for excitation stored in remote atomic ensembles. Nature
(London) 438, 828 (2005).

[14] Chou, C.-W. et al. Functional quantum nodes for entanglement distribution over scalable quantum networks. Science 316, 1316-1318 (2007).

[15] Jiang, L., Taylor, J. M. \& Lukin, M. D. Fast and robust approach to long-distance quantum communication with atomic ensembles. Phys. Rev. A 76, 012301 (2007).

[16] Foreman, S. M. et al. Coherent optical phase transfer over a $32-\mathrm{km}$ fiber with $1 \mathrm{~s}$ instability at $10^{-17}$. Phys. Rev. Lett. 99, 153601 (2007).

[17] Yuan, Z.-S. et al. Synchronized independent narrow-band single photons and efficient generation of photonic entanglement. Phys. Rev. Lett. 98, 180503 (2007).

[18] Moehring, D. L. et al. Entanglement of single-atom quantum bits at a distance. Nature (London) 449, 68-72 (2007).

[19] Wilk, T., Webster, S. C., Kuhn, A. \& Rempe, G. Singleatom single-photon quantum interface. Science $\mathbf{3 1 7}$, 488-490 (2007).

[20] Chen, Y.-A. et al. Memory-built-in quantum teleportation with photonic and atomic qubits. Nature Physics 4, 103-107 (2008).

[21] Chen, S. et al. A stable atom-photon entanglement source for quantum repeaters. Phys. Rev. Lett. 99, 180505 (2007).

[22] Kwiat, P. G. et al. New high-intensity source of polarization-entangled photon pairs. Phys. Rev. Lett. 75, 4337-4341 (1995).

[23] Pan, J.-W. \& Zeilinger, A. Greenberger-horne-zeilingerstate analyzer. Phys. Rev. A 57, 2208-2211 (1998).

[24] Harber, D. M., Lewandowski, H. J., McGuirk, J. M. \& Cornell, E. A. Effect of cold collisions on spin coherence and resonance shifts in a magnetically trapped ultracold gas. Phys. Rev. A 66, 053616 (2002).

[25] Kuga, T. et al. Novel optical trap of atoms with a doughnut beam. Phys. Rev. Lett. 78, 4713-4716 (1997).

[26] Simon, J., Tanji, H., Thompson, J. K. \& Vuletić, V. Interfacing collective atomic excitations and single photons. Phys. Rev. Lett. 98, 183601 (2007).

[27] Sangouard, N. et al. Robust and efficient quantum repeaters with atomic ensembles and linear optics. Phys. Rev. A 77, 062301 (2008).

Acknowledgements We thank Wolfgang Dür for discussion. This work was supported by the Deutsche Forschungsgemeinschaft, the Alexander von Humboldt Foundation, and the European Commission through the Marie Curie Excellence Grant and the ERC Grant. This work was also supported by the National Fundamental Research Program (Grant No.2006CB921900), the CAS, and the NNSFC.

Author Information The authors declare no competing financial interests. Correspondence and requests for materials should be addressed to Y.A.C (yuao@physi.uni-heidelberg.de) and J.W.P (jianwei.pan@physi.uni-heidelberg.de).

Online supplementary information http://www.nature.com/nature/journal/v454/n7208/ suppinfo/nature07241.html 